\title{
Improved geodetic European very-long-baseline interferometry solution using models of antenna gravitational deformation
}

\author{
Pierguido Sarti ${ }^{1,}{ }^{\star}$, Monia Negusini ${ }^{1}$, Claudio Abbondanza ${ }^{1}$ \\ ${ }^{1}$ Istituto di Radioastronomia (IRA) - Istituto Nazionale di Astrofisica (INAF), Bologna, Italy
}

\author{
Article history \\ Received May 24, 2010; accepted September 27, 2010. \\ Subject classification: \\ Crustal deformations, Measurements and monitoring, Theory and models, Instruments and techniques, Data processing.
}

\section{ABSTRACT}

Very-long-baseline interferometry (VLBI) is used for establishing global geodetic networks where the coordinates attain a 1-mm level of precision. Technique-dependent bias can degrade the VLBI positioning accuracy if it is present and unaccounted for. Among the potential bias, gravitational flexure of VLBI telescopes can vary the path traveled by the incoming radio signal and induce a bias in the height component of the station position. We process here more than 100 European VLBI sessions spanning 1990-2009 with VLBI time delay/Solve software, as the only VLBI analysis package that can be used to correct signal-path variation (SPV) due to gravitational flexure of VLBI telescopes. Currently, SPV models are neglected in VLBI data analysis. To determine the kinematics of the European area over the last 20 years and to assess the effects of telescope gravitational deformation on geodetic VLBI estimates, we perform two VLBI solutions with and without SPV models for telescopes in Medicina (northern Italy) and Noto (southern Italy). The two solutions differ by $8.8 \mathrm{~mm}$ and $7.2 \mathrm{~mm}$ in their height components, with this bias being one order of magnitude larger than the formal errors of the estimated heights. SPV models impact uniquely on the height component of stations where SPVs are modeled. Velocities are not affected by the use of the Medicina and Noto SPV models, and we show that the crustal kinematics derived from VLBI does not suffer from a lack of information with regard to the flexure of other telescopes.

\section{Introduction}

Since the 1970s, geodetic very-long-baseline interferometry (VLBI) has been used as a very precise global technique to investigate different geodetic and geophysical phenomena [see e.g. Robertson 1991]. In particular, in the late 1970s, the United States of America National Aeronautics and Space Administration (NASA) promoted the Crustal Dynamics Project, where VLBI was used in conjunction with satellite laser ranging (SLR) to investigate: (i) regional deformation and strain accumulation related to earthquakes at the western plate boundary in the USA; (ii) relative plate tectonic motions; (iii) internal deformation of lithospheric plates away from plate boundaries; (iv) polar motion and variations in Earth rotation, and their possible correlation with earthquakes and other geophysical phenomena; and (v) crustal motion and deformation that occurs in regions of high earthquake activity [Ryan et al. 1993].

The potential of the VLBI technique for addressing questions in Earth sciences and astrophysics attracted the interest of the Italian Consiglio Nazionale delle Ricerche (CNR). In the mid-1970s, the CNR designed and initiated the Italian VLBI project, with the dual purpose of developing national radio-astronomical facilities and extending the international VLBI geodetic network in the Mediterranean area for local and global geodynamic investigations. The construction of three identical VLBI radio telescopes in different parts of Italy was initially planned. However, the project underwent drastic financial cutbacks, which resulted in cancellation of the planned radio telescope in Sardinia [Setti 2006]. The VLBI radio telescopes in Medicina (northern Italy) and Noto (southern Italy) were built and completed in the 1980s. They are twin, 32-m, steerable, azimuth-elevation mount radio telescopes, and they have dual reflector systems with a Cassegrainian secondary focus configuration. Geodetic observations are performed at 2.3 $\mathrm{GHz}$ and $8.3 \mathrm{GHz}$, and the corresponding $\mathrm{S}$ and $\mathrm{X}$ receivers are located at the primary focus position.

The first geodetic observing session took place in January 1987 in Medicina [Tomasi et al. 1988], while the Noto geodetic observations began in June 1989 [Tomasi 1993]. These telescopes are now managed by the Istituto Nazionale di Astrofisica (INAF) through its department at the Istituto di Radioastronomia (IRA), which was formerly part of the CNR. Nowadays, both observatories are international terrestrial reference frame (ITRF) co-location sites, and they have a well-established, double co-location between VLBI and global positioning system (GPS) techniques. The tie vectors between the space geodetic instruments at both sites have been surveyed and rigorously estimated several times [Sarti et al. 2004], and they have served as benchmarks for the assessment of the reliability of different approaches for surveying tie vectors in ITRF computation [Abbondanza et 
al. 2009]. Co-location of geodetic instruments with longrunning observations, along with accurately repeated tie vectors, boosts the importance of geodetic infrastructures and their potential in geodetic research.

In 1990, coordinated observing sessions of the European VLBI network were regularly planned and carried out (EUROPE-VLBI sessions), with the purpose of investigating the deformation of the crust and vertical motion rates at a precision of a one-millimeter-per-year level [see e.g. Tomasi et al. 1999, Campbell and Nothnagel 2000]. Since 1990, 102 EUROPE-VLBI sessions have been performed regularly, with their observations correlated in Bonn (Germany).

VLBI collects the signals of extra-galactic radio sources, which provide the best possible approximation to a quasiinertial reference system, this being the best system for the investigation of Earth motion. Therefore, VLBI observations can be used to accurately measure and study the long and short term fluctuations of the orientation of the Earth. As a consequence, polar motion, UT1 and nutation are regularly investigated and accurately determined with VLBI, and they represent official International VLBI Service (IVS) products that are disseminated by the IVS Analysis Coordinator [Böckmann et al. 2010b].

VLBI is equally important for the definition and maintenance of global reference frames. Indeed, observations of extra-galactic radio-sources define the international celestial reference frame (ICRF). The recent resolution of the International Astronomical Union (IAU) states that since January 1,2010 , the fundamental astrometric realization of the International Celestial Reference System (ICRS) will be the second realization of the International Celestial Reference Frame (ICRF2). This is as constructed by the International Earth rotation and Reference system Service (IERS) and IVS [Schlüter and Behrend 2007] working group on the ICRF, in conjunction with the IAU Division I working group on ICRF2 [Fey et al. 2009]. VLBI also has a key role in the definition of the ITRF, as its frame scale contributes to the definition of the scale of the global combined frame [see e.g. Altamimi et al. 2007].

As far as crustal motion is concerned, global navigation satellite systems (GNSS) represent the most versatile, flexible and economic tools for monitoring deformation at global, regional and local scales. Nevertheless, VLBI maintains an important role in monitoring global tectonic processes, as well as in determining motion and deformation of the continental-scale plates that form the Earth crust. EUROPEVLBI sessions are an example of this latter application.

\section{Antenna near-field effects: height and scale bias}

A major limiting factor in the accuracy of space geodetic techniques is related to systematic errors of a different nature. In particular, near-field effects impact on the station position and they are often difficult to identify and mitigate.
Technique-specific bias thus prevents the highest level of accuracy in geodetic data processing.

Examples that can affect the position of space geodetic instruments include range bias for the SLR technique [see e.g. Coulot et al. 2008], satellite phase center motion in the Doppler Orbitography and Radioposition Integrated by Satellite (DORIS) technique [see e.g. Willis et al. 2007], and near-field effects for GNSS [see e.g. Dilssner et al. 2008]. Specifically, multipaths that originate in the near-field of GNSS antennas, uncalibrated antennas and uncalibrated radomes are nowadays an issue for $20 \%$ of the permanent sites of the International GNSS Service (IGS) [Dow et al. 2009] network and particularly problematic at ITRF colocation sites [King et al. 2010, Schmid et al. 2010]. These factors have unpredictable effects on the height component of the antenna reference point, and they originate problems in the maintenance of the IGS frame and contribute to the discrepancies, which stem from the combination of technique-specific frames.

Analogously, since the very beginning of VLBI observations, gravitational deformation of the radio telescope structures was recognized as a potentially important error source. Gravity-induced deformation changes the path length of the incoming radio signal in the near-field of the VLBI antenna as a function of the pointing elevation. A remarkable example of the effect of gravitational flexure on VLBI positioning was offered by the prescient work of Carter et al. [1980]. Comparing results obtained from ground surveys and VLBI data analysis, they found a $19-\mathrm{mm}$ difference in the vertical component of the 1.24-km-long Haystack-Westford baseline. Most of this difference $(13 \mathrm{~mm})$ was ascribed to the gravitational flexure of the 37-m Haystack telescope structure that was found to vary as the sine of the pointing elevation angle. The signature of this error aliases indistinguishably into the estimated VLBI height, thus biasing the relative VLBI antenna reference-point position. Since Carter et al. [1980], little additional work was done. Clark and Thomsen [1988] investigated and set-up the analytical approach to SPV model computation for primary focus VLBI telescopes. They carried out a detailed study on the 22-m, X-Y mount telescope in Fairbanks (Alaska, USA), using a finite element model (FEM) and a comprehensive geometric approach to combine the gravitational deformation and define the elevation-dependent SPV model. In their specific case, the near-field contribution to the SPV due to gravitational deformation was found to be negligible within the whole $\left[0^{\circ}\right.$, $90^{\circ}$ ] elevation range. This evidence turned out to be misleading, since it was believed to represent the deformational behavior of a wider range of telescopes [see e.g. Gendt et al. 2007]. This latter belief held for a couple of decades after Clark and Thomsen [1988] and discouraged further effective investigations into gravity-induced near-field 


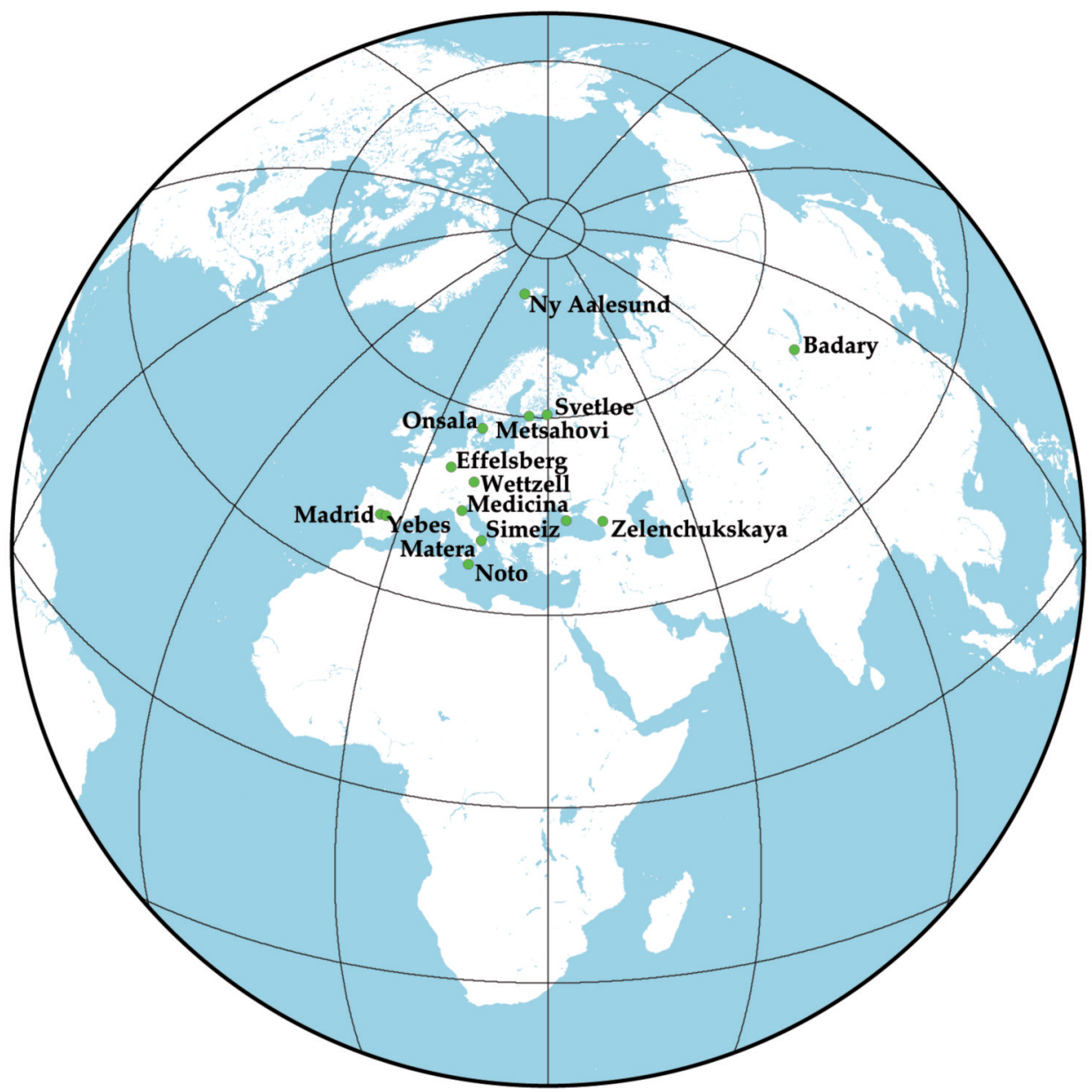

Figure 1. The European VLBI network currently operating in the framework of EUROPE-VLBI sessions.

SPVs. Only recently has research in this field flourished again, as the requirement for the greatest level of accuracy of geodetic products has increased.

\subsection{Signal-path variations in VLBI telescopes}

Sarti et al. [2009a] determined the SPV due to elevationdependent gravitational deformation of the Medicina and Noto telescopes using a combination of terrestrial surveying methods. Gravitational flexures of VLBI telescopes depend on the pointing elevation, $e$, of the antenna, relative to which they vary accordingly. They induce a variation $\Delta L(e)$ in the path traveled by the incoming radio signal in the nearfield of a VLBI telescope. This can be determined either with a FEM [Clark and Thomsen 1988] or with a combination of terrestrial methods [Sarti et al. 2009a]. $\Delta L(e)$ is expressed as a linear combination of three terms: (i) the variation of the focal length of the paraboloidal primary reflector, $\Delta F(e)$; (ii) the displacement of the vertex of the primary reflector along the line of sight, $\Delta V(e)$; and (iii) the displacement of the feed horn phase center (or the subreflector for secondary focus configuration layout) along the same direction, $\Delta R(e)$. This last term is directly related to the gravitational deformation of the quadrupode legs. The displacement of the vertex is related to the sag of the optical system under the effect of gravity. $\Delta F(e)$ is caused by the inward folding of the primary reflector as the pointing elevation decreases from zenith to horizon [Sarti et al. 2009b]. For radio telescopes with geodetic S/X band receivers located at the primary focus, the SPV is:

$$
\Delta L^{\prime}(e)=\alpha_{F}^{\prime} \Delta F(e)+\alpha_{V}^{\prime} \Delta V(e)+\alpha_{R}^{\prime} \Delta R(e) .
$$

When geodetic observations are performed in secondary focus, the SPV is expressed as [Abbondanza and Sarti 2010]:

$$
\Delta L^{\prime \prime}(e)=\alpha_{F}^{\prime \prime} \quad \Delta F(e)+\alpha_{V}^{\prime \prime} \quad \Delta V(e)+2 \alpha_{R}^{\prime \prime} \quad \Delta R(e) .
$$

The linear coefficients $\left(\alpha_{F}^{\prime}, \alpha_{F}^{\prime \prime}\right)$ are the partial derivatives of the SPV with respect to the focal-length variation and $\left(\alpha_{V}^{\prime}, \alpha_{V}^{\prime \prime}, \alpha_{R}^{\prime}, \alpha_{R}^{\prime \prime}\right)$ are the partial derivatives with respect to the vertex and receiver (or sub-reflector) displacements, respectively [Sarti et al. 2009a]. Details of the analytical derivation of Equations (2.1.1) and (2.1.2) and of the rigorous computation of the linear coefficients $\alpha_{i}^{j}$ can be found in Abbondanza and Sarti [2010].

Contrary to the Fairbanks antenna example, the SPV for the Medicina and Noto $32-\mathrm{m}$ telescopes is almost $1 \mathrm{~cm}$. The effect of gravity-related SPV on geodetic VLBI data analysis was recently investigated by Sarti et al. [2010], who focused on 
the differences between the estimated geodetic parameters obtained with and without SPV models. Using the whole set of VLBI observations up to December 2009, Sarti et al. [2010] show that for the Medicina and Noto telescopes, the SPV corrections are one order of magnitude greater than the VLBI formal error, and they uniquely bias the height component of the telescope reference point. Therefore, gravitational deformation reduces the accuracy of VLBI positioning and questions the reliability of its frame scale.

Prior to Sarti et al. [2010], no quantitative analysis of the relationship between telescope gravitational flexure and VLBI frame scale had been carried out. It is easy to understand how important the consequences are for the ITRF computation when considering how its scale is defined in the combination of each technique-specific frame [Altamimi et al. 2007, Altamimi 2010, Angermann et al. 2010].

Sarti et al. [2010] show that SPV models are mandatory to pursue an utmost level of accuracy. Therefore, SPV models must be computed for every telescope of the IVS network, and as they become available, they must be used in the VLBI data processing.

\section{Data processing}

In the present study, we considered the whole VLBI dataset of 102 EUROPE-VLBI sessions that have been acquired since 1990, and using VLBI time delay/Solve [Petrov 2008], we performed two different VLBI solutions. One solution, $R$, can be regarded as routine processing according to the IVS standards and it does not introduce any information about telescope gravitational flexure. The other solution, $G$, applies the same set of models and parameters, except for the SPV models for the Medicina and Noto telescopes. In the next section, we discuss the differences between the solutions $R$ and $G$, and we provide an updated sketch of the velocity field in the European area, as derived using the VLBI technique. The European geodetic VLBI network now comprises a total of 14 sites (Figure 1).

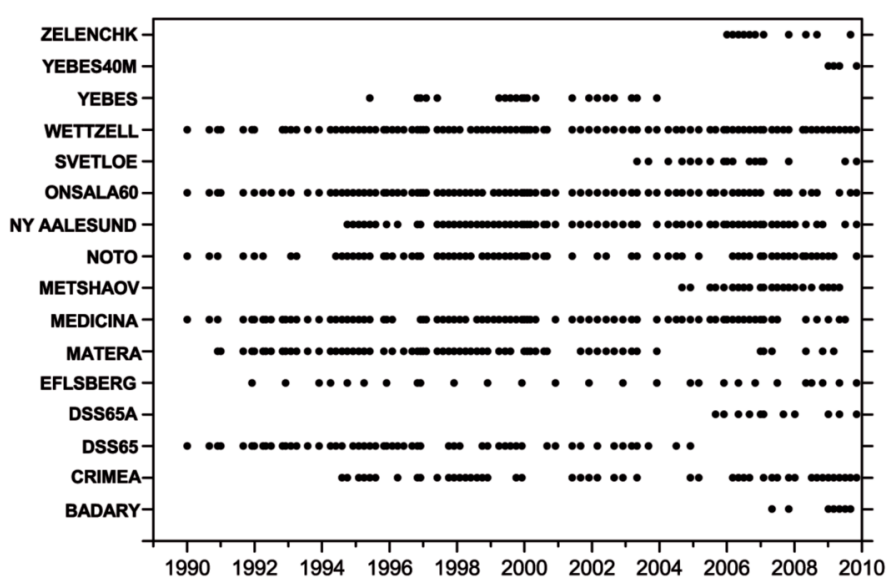

Figure 2. Participation of the VLBI stations in EUROPE-VLBI sessions. Black dots, sessions in which the VLBI stations participated.
The early 1990s sessions also involved mobile VLBI equipment in Karlsburg (Germany) and Toulouse (France), as well as the additional mobile telescope of TIGO in Wettzell (Germany), which has been moved and is now operating in Concepcion (Chile) as TIGOCONC. In Yebes (Spain), a brand new 40-m, azimuth-elevation radio telescope replaced the smaller and older 14-m antenna in 2008. Finally, the DSS65 radio telescope in Madrid (Spain) was moved in 2005 to a nearby location, and adopted the new code DSS65A. For both of the Spanish telescopes, the velocities of the new antennas are constrained to be equal to those of the former ones. EUROPE-VLBI sessions have been regularly carried out over two decades, with the coordinated effort of the radio astronomical European facilities. The most important VLBI stations and their participation in the EUROPE-VLBI sessions are shown in Figure 2.

The 102 24-hour VLBI European sessions were reduced with analysis software VLBI time delay/Solve [Petrov 2008], a modern extension of the popular CALC/SOLVE package that was developed at NASA in the 1970s. A detailed description of a reduction model, parameterization and constraints that are applied in geodetic VLBI data analysis can be found in Petrov et al. [2009]. We estimated mean site positions, linear velocities, harmonic site position variations, zenith path delay in the neutral atmosphere, atmospheric gradients, and station clock function represented with a Bspline. Source coordinates and Earth orientation parameters were not estimated: the corresponding IERS product values were used as reference in the solutions. Antenna thermal deformations, i.e. the motions of the telescope reference point due to thermal expansion of the structure of the antenna, were modeled according to the recent IVS guidelines [Nothnagel 2008]. The axis off-set of the telescopes is not estimated, but is fixed to the IVS values. Both solutions $R$ (no correction of gravitational flexure) and $G$ (correction of gravitational flexure for the Medicina and Noto telescopes) were transformed into ITRF2005 by applying a no-net-rotation and no-net-translation condition to the ITRF2005 coordinates of the Wettzell, Ny Ålesund, Madrid, Matera and Onsala stations. Furthermore, the Wettzell and Onsala velocities were constrained to the corresponding ITRF2005 values. The a-priori coordinates of the stations of the sub-network are equal in both the $R$ and $G$ runs. The no-net-translation condition maintains the barycenter of the five station sub-network as unaltered, and it is useful to directly assess the impact of the SPV models on the coordinates of the Medicina and Noto telescopes, as these sites are excluded from the no-net-translation constraint.

Equations (2.1.1) and (2.1.2) model the SPV as a function of the pointing elevation in primary and secondary focus radio telescopes, respectively, and must be used in the data analysis to account for and correct the bias of the VLBI 


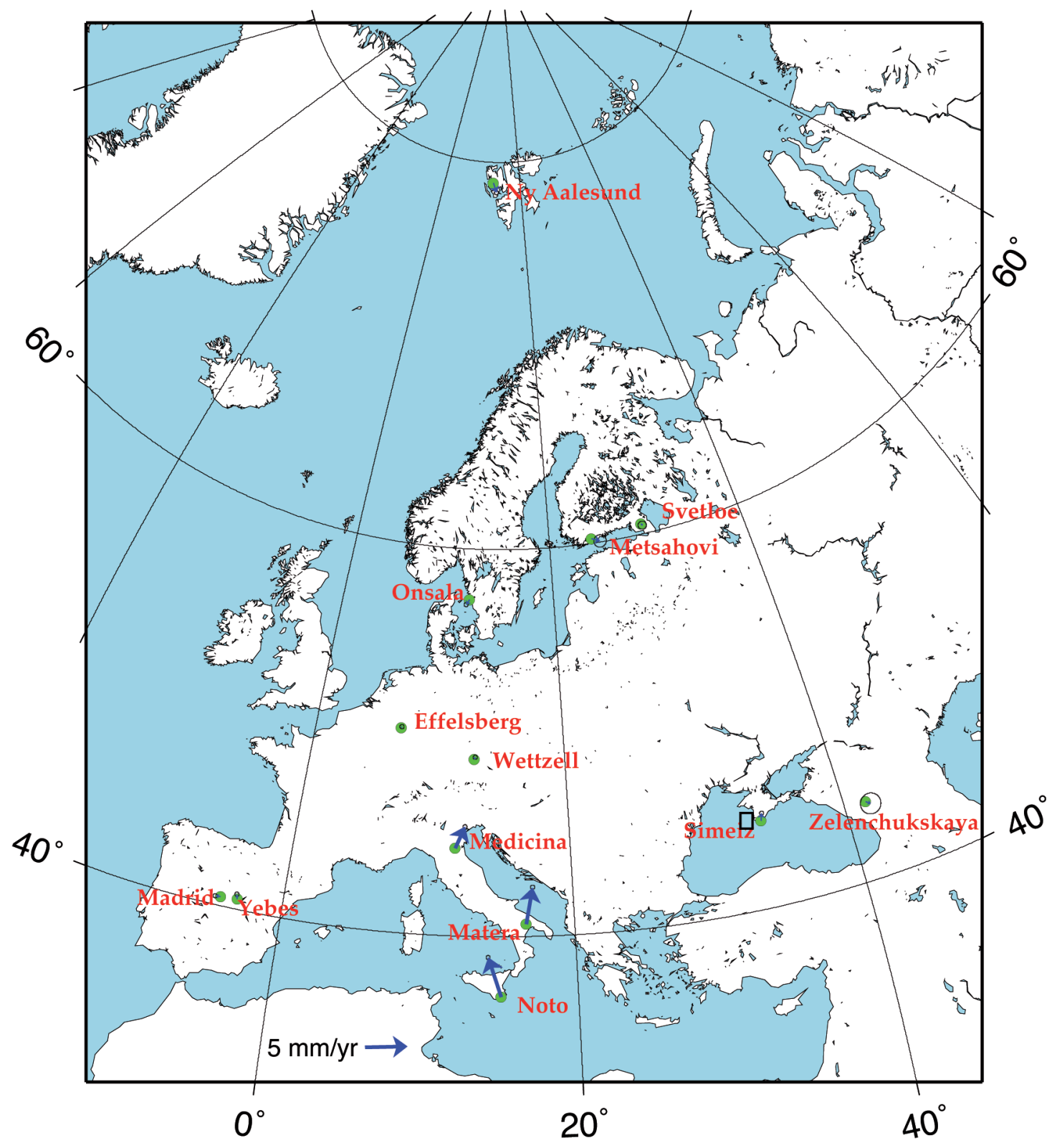

Figure 3. Horizontal velocities of the European sites obtained from the $G$ solution and expressed in the corresponding local topocentric frames. The velocities are relative to the ITRF2005 Eurasian plate motion: the Euler pole location and its rotation rate [Altamimi et al. 2007] have been subtracted from the estimated velocities to derive relative deformation rates at each site. The corresponding values are in Table 1, columns 9 and 10.

delay induced by gravitational flexure. As shown by Sarti et al. [2010], it is impossible to discover and correct this bias using only VLBI observations. Therefore, site-specific SPV models are strictly necessary for every VLBI telescope, to ensure unbiased VLBI estimates.

SPV files can be regarded as being analogous to absolute phase center variation files in GNSS antennas, since both files model the electronic reference-point motion with respect to the conventional reference point [Sarti et al. 2009a]. It is very well known that poor definition of phase center variation files dramatically impacts upon the accuracy and reliability of GNSS results.

Nowadays, the only operating telescopes of the IVS network with SPV models are those of Medicina and Noto. In particular, the SPV model for the Medicina telescope is expressed by:

$$
\Delta L_{M d}=0.22 \cdot e-0.0012 \cdot e^{2}
$$

and the SPV model for the Noto telescope is:

$$
\Delta L_{N t}=0.12 \cdot e-0.00045 \cdot e^{2}
$$

where $\Delta L_{M d}$ and $\Delta L_{N t}$ are expressed in $(\mathrm{mm})$ and the elevation $e$ over the range $\left[0^{\circ}, 90^{\circ}\right]$. The SPVs are modeled by a monotonic second-order function for which the minimum value is $0 \mathrm{~mm}$ in $0^{\circ}$ and the maximum values are $\Delta L_{M d}^{\max }(90)=10.1 \mathrm{~mm}$ and $\Delta L_{N t}^{\max }(90)=7.2 \mathrm{~mm}$. Details of the derivation of Equations (3.1) and (3.2) can be found in Abbondanza and Sarti [2010].

The lack of SPVs for other telescopes means that at present routine VLBI solutions do not take into account gravitational flexure of the antenna structures. Therefore, if 


\begin{tabular}{|c|c|c|c|c|c|c|c|c|c|}
\hline \multirow[t]{2}{*}{ Site } & \multicolumn{4}{|c|}{ Absolute velocity vectors } & \multirow{2}{*}{$\begin{array}{c}\text { Azimuth } \\
\text { (deg) }\end{array}$} & \multicolumn{3}{|c|}{$\begin{array}{c}\text { Eurasian plate adjusted } \\
\text { velocity vectors }\end{array}$} & \multirow{2}{*}{$\begin{array}{c}\text { Azimuth rel. } \\
\text { (deg) }\end{array}$} \\
\hline & $\underset{(\mathbf{m m} / \mathbf{a})}{\mathrm{Up}}$ & $\begin{array}{c}\text { East } \\
(\mathbf{m m} / \mathbf{a})\end{array}$ & $\begin{array}{l}\text { North } \\
(\mathrm{mm} / \mathbf{a})\end{array}$ & $\begin{array}{l}\text { Horiz. } \\
(\mathrm{mm} / \mathrm{a})\end{array}$ & & $\begin{array}{l}\text { East rel. } \\
(\mathbf{m m} / \mathbf{a})\end{array}$ & $\begin{array}{l}\text { North rel. } \\
(\mathbf{m m} / \mathbf{a})\end{array}$ & $\begin{array}{c}\text { Horiz. rel. } \\
(\mathbf{m m} / \mathbf{a})\end{array}$ & \\
\hline Badary & $7.5 \pm 5.6$ & $25.3 \pm 1.3$ & $-5.7 \pm 1.1$ & $25.9 \pm 1.4$ & $102.8 \pm 2.2$ & $-1.7 \pm 1.3$ & $-0.7 \pm 1.1$ & $1.8 \pm 1.4$ & $247.0 \pm 2.2$ \\
\hline Simeiz & $1.7 \pm 0.3$ & $24.7 \pm 0.1$ & $13.2 \pm 0.1$ & $28.0 \pm 0.1$ & $62.0 \pm 0.1$ & $0.2 \pm 0.1$ & $0.9 \pm 0.1$ & $0.9 \pm 0.1$ & $15.3 \pm 0.1$ \\
\hline Madrid & $3.3 \pm 0.1$ & $18.2 \pm 0.1$ & $16.1 \pm 0.1$ & $24.3 \pm 0.1$ & $48.5 \pm 0.1$ & $-0.6 \pm 0.1$ & $0.0 \pm 0.1$ & $0.6 \pm 0.1$ & $270.0 \pm 0.1$ \\
\hline Effelsberg & $3.2 \pm 0.1$ & $18.2 \pm 0.1$ & $15.8 \pm 0.1$ & $24.1 \pm 0.1$ & $49.0 \pm 0.1$ & $0.0 \pm 0.1$ & $0.1 \pm 0.1$ & $0.1 \pm 0.1$ & $5.2 \pm 0.1$ \\
\hline Matera & $0.7 \pm 0.1$ & $23.2 \pm 0.1$ & $19.2 \pm 0.1$ & $30.1 \pm 0.1$ & $50.5 \pm 0.1$ & $0.8 \pm 0.1$ & $4.3 \pm 0.1$ & $4.4 \pm 0.1$ & $10.8 \pm 0.1$ \\
\hline Medicina & $-1.2 \pm 0.1$ & $21.7 \pm 0.1$ & $17.9 \pm 0.1$ & $28.2 \pm 0.1$ & $50.5 \pm 0.1$ & $1.1 \pm 0.1$ & $2.6 \pm 0.1$ & $2.8 \pm 0.1$ & $22.2 \pm 0.1$ \\
\hline Metshaovi & $3.0 \pm 1.3$ & $20.1 \pm 0.3$ & $13.4 \pm 0.4$ & $24.2 \pm 0.3$ & $56.2 \pm 0.8$ & $1.0 \pm 0.3$ & $-0.4 \pm 0.4$ & $1.1 \pm 0.3$ & $113.4 \pm 0.8$ \\
\hline Noto & $-0.1 \pm 0.1$ & $21.3 \pm 0.1$ & $19.7 \pm 0.1$ & $29.0 \pm 0.1$ & $47.1 \pm 0.1$ & $-1.5 \pm 0.1$ & $4.7 \pm 0.1$ & $5.0 \pm 0.1$ & $341.8 \pm 0.1$ \\
\hline Ny Ålesund & $7.6 \pm 0.1$ & $9.9 \pm 0.1$ & $14.2 \pm 0.1$ & $17.3 \pm 0.1$ & $34.9 \pm 0.1$ & $0.4 \pm 0.1$ & $-1.1 \pm 0.1$ & $1.2 \pm 0.1$ & $160.6 \pm 0.1$ \\
\hline Onsala & $3.7 \pm 0.1$ & $16.9 \pm 0.1$ & $14.7 \pm 0.1$ & $22.3 \pm 0.1$ & $49.0 \pm 0.1$ & $-0.4 \pm 0.1$ & $-0.6 \pm 0.1$ & $0.7 \pm 0.1$ & $209.3 \pm 0.1$ \\
\hline Svetloe & $1.1 \pm 0.7$ & $20.2 \pm 0.2$ & $12.8 \pm 0.2$ & $23.9 \pm 0.2$ & $57.6 \pm 0.1$ & $0.1 \pm 0.2$ & $-0.2 \pm 0.2$ & $0.3 \pm 0.2$ & $151.7 \pm 0.1$ \\
\hline Wettzell & $0.4 \pm 0.1$ & $19.9 \pm 0.1$ & $15.5 \pm 0.1$ & $25.2 \pm 0.1$ & $52.1 \pm 0.1$ & $0.1 \pm 0.1$ & $0.3 \pm 0.1$ & $0.3 \pm 0.1$ & $17.7 \pm 0.1$ \\
\hline Yebes & $3.4 \pm 0.1$ & $18.8 \pm 0.1$ & $16.6 \pm 0.1$ & $25.1 \pm 0.1$ & $48.5 \pm 0.1$ & $-0.2 \pm 0.1$ & $0.5 \pm 0.1$ & $0.5 \pm 0.1$ & $343.6 \pm 0.1$ \\
\hline Zelenchukskaya & $14.1 \pm 2.5$ & $25.2 \pm 0.5$ & $10.5 \pm 0.5$ & $27.3 \pm 0.5$ & $67.5 \pm 1.0$ & $-0.5 \pm 0.5$ & $-0.4 \pm 0.5$ & $0.6 \pm 1.0$ & $231.0 \pm 1.0$ \\
\hline
\end{tabular}

Table 1. Velocities of the European stations expressed in the topocentric frame at each site. Columns 3 and 4: components of the absolute horizontal velocity vector, for which the magnitude is in column 5 and the direction in column 6. Columns 7-10: analogous to previous quantities but with the rigid rotation of the Eurasian plate subtracted. Column 2 and columns 9 and 10: data used in Figures 4 and 3, respectively.

this flexure is present, elevation-dependent signal-path delay bias induced by gravity cannot be removed. This is the case for the official IVS solution supplied to the IERS combination centers for the computation of the ITRF [see e.g. Böckmann et al. 2010a].

We compute a routine solution, $R$, where gravitational flexures are disregarded, according to the usual processing policy of the IVS. Another solution $G$ is computed, which introduces the SPV models for the Noto and Medicina telescopes as expressed by Equations (3.1) and (3.2).

\section{Results}

A comparison between the estimated local geodetic coordinates derived by EUROPE-VLBI sessions in solutions $R$ and $G$ shows no variations for all stations of the European network except for the Medicina and Noto up component. In particular, the upward shift that originates from the use of SPV models is $8.8 \mathrm{~mm}$ for Medicina and $7.2 \mathrm{~mm}$ for Noto. This confirms the behavior that was first described by Sarti et al. [2010], who processed the whole dataset of geodetic VLBI observations: in that case, the application of SPV models to the Medicina and Noto stations resulted in an upward shift of $8.9 \mathrm{~mm}$ and $6.7 \mathrm{~mm}$.

In terms of velocities, solutions $R$ and $G$ do not show any significant difference and SPV models impact on solution
$G$, removing from the station height the almost constant bias due to gravitational flexures.

Horizontal velocities of the European VLBI sites are shown in Figure 3. For the benefit of clarity, Badary is not shown in Figure 3, as the longitude of this site is very different from those of the other stations.

The ITRF2005 Euler pole of the Eurasian plate [Altamimi et al. 2007] was subtracted from the estimated absolute local velocities, to derive information on the regional kinematic field. Horizontal relative velocities are expressed in the local geodetic frames, and the corresponding values are reported in Table 1, columns 9 and 10.

The horizontal motions of almost all of the sites are well within $1.5 \mathrm{~mm} / \mathrm{a}$, with all central European sites showing relative velocities of a few tenths of a $\mathrm{mm} / \mathrm{a}$ (Table 1, column 9). Exceptions are seen for the Italian VLBI sites, the relative velocities of which are between 2.5 and 5.0 $\mathrm{mm} / \mathrm{a}$. Medicina and Matera move towards the NNE at 2.8 $\mathrm{mm} / \mathrm{a}$ and $4.4 \mathrm{~mm} / \mathrm{a}$, respectively. The Noto station shows a $5.0 \mathrm{~mm} /$ a NNW motion with respect to the Eurasian plate.

Vertical velocities are shown in Figure 4 and the corresponding values are reported in Table 1, column 2. As previously stated, the $R$ and $G$ solutions are totally consistent in terms of estimated velocities, and the values in Table 1 can be regarded as referred to either solution $R$ or $G$. 


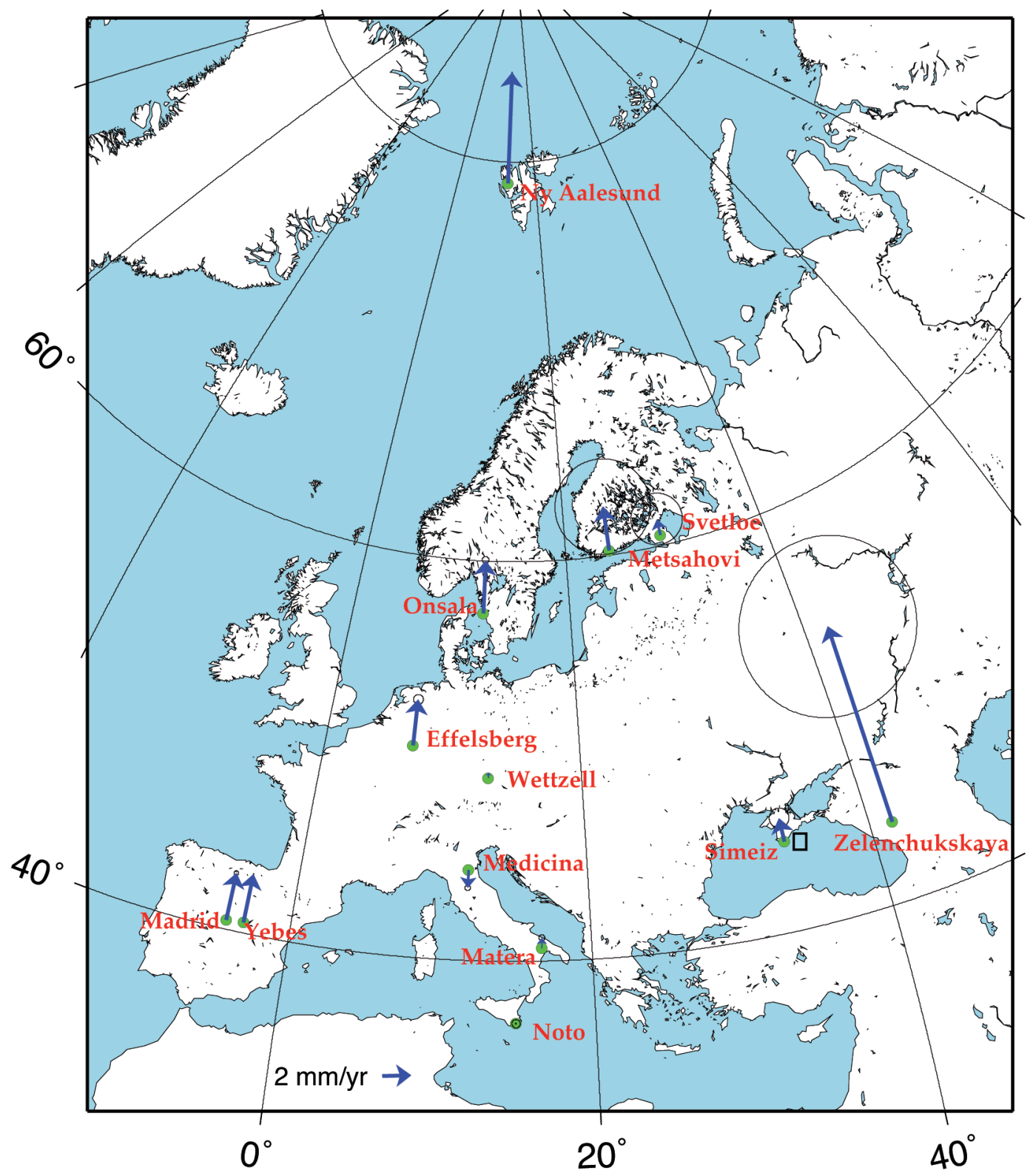

Figure 4. Local vertical velocities of the European VLBI sites (Table 1, column 2).

The uplift rates of Ny Ålesund in the Svalbard Islands, Badary and Zelenchukskaya (Russia) are almost twice the magnitude of those of the other stations. The Russian antennas have a rather short observation history, and their uplift rates still need to be confirmed by further observations. Conversely, the high uplift rate of $\mathrm{Ny}$ Ålesund $(7.6 \mathrm{~mm} / \mathrm{a})$ is more than double the uplift of the other European sites, and it has been well determined from observations that have covered more than 16 years. It confirms the uplift rates due to the process of post-glacial rebound that is currently occurring in the area [see e.g. Kierulf et al. 2009]. The other stations in the Scandinavian area are Metsähovi (Finland), Svetloe (Russia) and Onsala (Sweden), and they are located in a region that is undergoing uplift due to glacial isostatic adjustment. In particular, Lidberg et al. [2010] determined a 3-D velocity field from a dataset of almost 10 years (up to early November 2006) of continuous observations carried out with a dense GPS network in Fennoscandia. It is worth highlighting that VLBI observations do not span the same time range. Observations in Svetloe and Metsähovi started in 2003 and 2004 and cover seven and six years, respectively. Onsala has been part of the EUROPE-VLBI sessions since 1990 and its observations span a 20-year period (see Figure 2). GPS-derived uplift rates [Lidberg et al. 2010] are larger than those derived by VLBI, and they differ by $2.2 \mathrm{~mm} / \mathrm{a}$ at Metsähovi, $2.0 \mathrm{~mm} / \mathrm{a}$ at Svetloe, and $0.3 \mathrm{~mm} / \mathrm{a}$ at Onsala.

Medicina is the only European site that shows a small, although significant, negative linear trend of $-1.2 \mathrm{~mm} / \mathrm{a}$ over the period 1990-2009. When compared to previous determinations [see e.g. Bitelli et al. 2005], the velocity appears to have diminished. A decrease in the subsidence rate at Medicina is confirmed by gravity and GPS observations, and it is apparently related to fluctuations in local hydrological conditions associated with climate variations [Zerbini et al. 2010]. 


\begin{tabular}{|c|c|c|c|c|c|c|}
\hline BASELINE & \begin{tabular}{|l|}
$\begin{array}{l}\text { Length } \\
\text { (mm) }\end{array}$ \\
\end{tabular} & $\begin{array}{l}\text { St. dev. } \\
(\mathrm{mm})\end{array}$ & $\begin{array}{l}\text { Velocity } \\
(\mathrm{mm} / \mathrm{a})\end{array}$ & $\begin{array}{l}\text { St. dev. } \\
(\mathrm{mm} / \mathrm{a})\end{array}$ & $\begin{array}{l}\text { G-R } \\
(\mathbf{m m})\end{array}$ & \# sess \\
\hline Effelsberg/Madrid & 1414346831.7 & 0.6 & 1.1 & 0.1 & - & 14 \\
\hline Effelsberg/Matera & 1333777522.4 & 0.5 & -2.8 & 0.0 & - & 16 \\
\hline Effelsberg/Medicina & 757049139.8 & 0.4 & -1.6 & 0.0 & 0.5 & 22 \\
\hline Effelsberg/Noto & 1644303648.2 & 0.4 & -4.4 & 0.0 & 0.9 & 16 \\
\hline Effelsberg/Ny Ålesund & 3140166753.5 & 0.5 & 1.5 & 0.1 & - & 20 \\
\hline Effelsberg/Onsala & 832210490.8 & 0.4 & -0.4 & 0.0 & - & 22 \\
\hline Effelsberg/Simeiz & 2129562246.7 & 0.7 & 0.6 & 0.1 & - & 12 \\
\hline Effelsberg/Wettzell & 457481743.9 & 0.3 & 0.1 & 0.0 & - & 26 \\
\hline Madrid/Matera & 1765812157.3 & 0.6 & 1.5 & 0.1 & - & 42 \\
\hline Madrid/Medicina & 1378852932.1 & 0.5 & 2.4 & 0.0 & 0.9 & 45 \\
\hline Madrid/Noto & 1711832875.6 & 0.4 & -2.0 & 0.0 & 1.0 & 37 \\
\hline Madrid / Ny Ålesund & 4264145228.7 & 0.6 & 2.8 & 0.0 & - & 79 \\
\hline Madrid/Onsala & 2205023118.9 & 0.6 & 0.7 & 0.1 & - & 49 \\
\hline Madrid/Wettzell & 1655418199.9 & 0.5 & 1.1 & 0.0 & - & 50 \\
\hline Madrid/Yebes & 99135252.1 & 0.5 & 0.6 & 0.0 & - & 8 \\
\hline Matera/Medicina & 597262269.0 & 0.4 & -1.6 & 0.0 & 0.4 & 57 \\
\hline Matera/Noto & 444532989.3 & 0.4 & 0.4 & 0.0 & 0.2 & 49 \\
\hline Matera/Ny Ålesund & 4190331410.9 & 0.5 & -2.4 & 0.0 & - & 39 \\
\hline Matera/Onsala & 1886809253.3 & 0.4 & -4.0 & 0.0 & - & 55 \\
\hline Matera/Wettzell & 990053311.1 & 0.4 & -3.6 & 0.0 & - & 62 \\
\hline Matera/Yebes & 1667614549.4 & 0.5 & 1.0 & 0.0 & - & 14 \\
\hline Medicina/Metsähovi & 1935196007.7 & 0.9 & -2.4 & 0.4 & 1.4 & 13 \\
\hline Medicina/Noto & 893724168.7 & 0.3 & -3.0 & 0.0 & 1.1 & 57 \\
\hline Medicina/Ny Ålesund & 3776620894.3 & 0.3 & -1.7 & 0.0 & 2.6 & 56 \\
\hline Medicina/Onsala & 1429470341.8 & 0.3 & -3.0 & 0.0 & 1.0 & 72 \\
\hline Medicina/Svetloe & 2139526939.2 & 0.7 & -2.9 & 0.2 & 1.5 & 15 \\
\hline Medicina/Wettzell & 522461078.3 & 0.2 & 3.0 & 0.0 & 0.4 & 79 \\
\hline Medicina/Yebes & 1285368221.6 & 0.4 & 10.8 & 0.0 & 0.9 & 16 \\
\hline Medicina/Zelenchuksk. & 2367843373.5 & 1.4 & 6.6 & 0.6 & 1.7 & 9 \\
\hline Metsähovi/Noto & 2660964212.3 & 1.0 & 7.3 & 0.4 & 1.5 & 14 \\
\hline Metsähovi/ Ny Ålesund & 2122206903.1 & 1.1 & 7.8 & 0.4 & - & 14 \\
\hline Metsähovi/Onsala & 784071663.5 & 0.8 & 4.3 & 0.3 & - & 13 \\
\hline Metsähovi/Svetloe & 299235376.0 & 0.9 & 3.2 & 0.4 & - & 8 \\
\hline Metsähovi/Wettzell & 1431538678.0 & 0.8 & 3.5 & 0.4 & - & 18 \\
\hline Metsähovi/Zelenchuksk. & 2150789456.0 & 1.6 & 10.9 & 0.7 & - & 6 \\
\hline Noto/Ny Ålesund & 4580537548.5 & 0.5 & 12.1 & 0.1 & 2.6 & 50 \\
\hline Noto/Onsala & 2280154795.5 & 0.4 & 6.6 & 0.0 & 1.2 & 62 \\
\hline Noto/Svetloe & 2808545447.1 & 0.8 & 10.2 & 0.2 & 1.6 & 10 \\
\hline Noto/Wettzell & 1371100965.9 & 0.3 & 5.6 & 0.0 & 0.7 & 69 \\
\hline Noto/Yebes & 1616522896.0 & 0.5 & 10.0 & 0.0 & 0.9 & 15 \\
\hline Noto/Zelenchukskaya & 2359618069.2 & 1.4 & 4.5 & 0.7 & 1.3 & 8 \\
\hline Ny Ålesund/ Onsala & 2387493150.8 & 0.3 & 7.3 & 0.0 & - & 60 \\
\hline Ny Ålesund/Svetloe & 2133123005.0 & 0.7 & 7.8 & 0.2 & - & 17 \\
\hline Ny Ålesund/Wettzell & 3283002125.3 & 0.2 & 9.8 & 0.0 & - & 66 \\
\hline Ny Ålesund/Yebes & 4246452742.1 & 0.7 & 14.7 & 0.0 & - & 15 \\
\hline Ny Ålesund/Zelenchuksk. & 4043126024.4 & 1.8 & 15.4 & 0.9 & - & 9 \\
\hline Onsala/Svetloe & 1079812940.5 & 0.6 & 7.0 & 0.2 & - & 14 \\
\hline Onsala/Wettzell & 919660985.5 & 0.3 & 3.2 & 0.0 & - & 85 \\
\hline Onsala/Yebes & 2153592386.9 & 0.5 & 8.9 & 0.0 & - & 17 \\
\hline Onsala/Zelenchukskaya & 2537588030.6 & 1.4 & 9.1 & 0.6 & - & 8 \\
\hline Simeiz/Madrid & 3117042125.0 & 0.9 & 1.9 & 0.1 & - & 17 \\
\hline Simeiz/Matera & 1472857706.7 & 0.6 & -1.7 & 0.1 & - & 22 \\
\hline Simeiz/Medicina & 1766185783.9 & 0.6 & -1.2 & 0.1 & 1.2 & 32 \\
\hline Simeiz/Metsähovi & 1866587991.7 & 1.1 & -0.9 & 0.4 & - & 12 \\
\hline Simeiz/Noto & 1798600353.3 & 0.6 & -0.6 & 0.1 & 1.0 & 32 \\
\hline Simeiz/Ny Ålesund & 3896968734.9 & 1.0 & 0.9 & 0.1 & - & 34 \\
\hline Simeiz/Onsala & 2093576822.6 & 0.7 & 0.3 & 0.1 & - & 34 \\
\hline Simeiz/Svetloe & 1810877604.5 & 0.9 & -0.7 & 0.2 & - & 8 \\
\hline Simeiz/Wettzell & 1684604515.5 & 0.6 & 0.1 & 0.1 & - & 39 \\
\hline Simeiz/Yebes & 3023893871.0 & 0.9 & 1.3 & 0.1 & - & 7 \\
\hline Simeiz/Zelenchukskaya & 610832160.4 & 1.2 & 0.2 & 0.5 & - & 8 \\
\hline Svetloe/Wettzell & 1654774847.5 & 0.6 & 6.6 & 0.2 & - & 18 \\
\hline Svetloe/Zelenchukskaya & 2014661043.3 & 1.4 & 14.0 & 0.6 & - & 6 \\
\hline Wettzell/Yebes & 1575653113.4 & 0.4 & 9.8 & 0.0 & - & 17 \\
\hline Wettzell/Zelenchukskaya & 2255828960.2 & 1.3 & 8.1 & 0.6 & - & 11 \\
\hline
\end{tabular}

Table 2. Baselines formed by the European IVS stations observed in more than five sessions (column 7). Baseline lengths (at epoch 01.01.2009) and their standard deviations, obtained from solution $G$, are shown in columns 2 and 3 . The rates of change of baselines lengths and their standard deviations are shown in columns 4 and 5. Column 6 contains the differences between the baselines lengths estimated using ( $G$ solution) and not using ( $R$ solution) SPV models. Zero differences are not reported. It is evident that introducing SPV models uniquely affects the baselines formed by stations being corrected for gravitational flexure. 
The modeling of gravitational flexure has a straightforward effect on the heights of the stations being corrected. Therefore, it reflects on the length component of all of the baselines involving telescopes where the deformations are modeled with an SPV. Table 2 lists the most significant baselines of the European VLBI network at epoch 01.01.2009. Table 2, column 4, shows their rates of change and Table 2, column 6 , shows the baseline length variations obtained with solutions $R$ and $G$. Their estimates vary uniquely if a SPV model is associated with one or both telescopes that form the baseline endpoints.

\section{Conclusions}

VLBI is a well-established space geodetic technique with over 30 years of continuous and precise observation records. Since 1990, dedicated VLBI measurements have been regularly carried out for monitoring the crustal kinematics in the European area with a sub-network of the permanent IVS network. The dataset is consistent and covers a longer time span than any other space geodetic technique.

Almost all Eurasian VLBI sites show a very high horizontal stability, with their horizontal velocities in the order of $1 \mathrm{~mm} / \mathrm{a}$ or less. Exceptions are represented by three Italian VLBI sites, at Matera, Medicina and Noto, with the last here being part of the African plate. Noto shows a velocity of $5.0 \mathrm{~mm} /$ a towards the NNW, which represents the highest value with respect to the ITRF2005 Eurasian Euler pole. For the vertical rates, Medicina is the only station that shows a statistically significant downward motion $(-1.2$ $\pm 0.1 \mathrm{~mm} / \mathrm{a})$. The Russian stations of Badary and Zelenchukskaya show a high uplift rate, the values of which need to be confirmed due to their short observation span. The Fennoscandian sites confirm the uplift rate related to post glacial rebound, although the VLBI rates are smaller than those derived in other studies using GPS.

Observation bias introduced by gravitational flexure of VLBI radio telescopes is not easy to mitigate, since this requires a complete and precise SPV model to account for the dependency of the radio signal phase on the elevation. The SPV model strictly depends on the design of the radio telescope. It can be defined by relying on ad-hoc terrestrial surveys and finite element models. When gravity-induced, elevation-dependent phase shifts are disregarded, the estimated height component of the antenna reference point can undergo unpredictably large bias. This reflects on the baselines lengths and the scale of the network.

For the specific cases of the Medicina and Noto telescopes, we show that in the analysis of purely European VLBI sessions, the upward shift of the height component is determined as $8.8 \mathrm{~mm}$ and $7.2 \mathrm{~mm}$, respectively. This bias systematically affects the position of the antenna reference point, although it does not affect the velocity estimates. This confirms that geodetic VLBI can be used to determine the intra-plate and the global kinematics, and these latter are not corrupted by the possible presence of gravitational flexure of radio telescopes.

The VLBI technique is confirmed as a reliable tool for studying the motion of the Earth crust over long time periods. Nevertheless, our results confirm the findings of Sarti et al. [2010], and they highlight the tight link between gravitational flexure and height component bias. Therefore, VLBI-derived absolute heights can be questioned in the absence of SPV models, and their use requires awareness and special care.

This limitation can be overcome by carrying out sitespecific surveys that are aimed at defining the SPV models for each VLBI telescope and by implementing these latter in routine VLBI data analysis. These are mandatory tasks for the short-term activity plan of the IVS, which must focus on this matter, and they comprise specific actions that are directed at mitigating gravitational flexure bias and improving the accuracy of the position and scale derived from VLBI.

Acknowledgements. The authors thank Tonie van Dam for her careful review and improvements to the text. This study is based on observations acquired with the Medicina and Noto radio telescopes operated by INAF - IRA. All of the maps were made with the General Mapping Tool (GMT) software [Wessel and Smith 1998], which is available at http://gmt.soest.hawaii.edu under the GNU General Public License.

\section{References}

Abbondanza, C., Z. Altamimi, P. Sarti, M. Negusini and L. Vittuari (2009). Local effects of redundant terrestrial and GPS-based tie vectors in ITRF-like combinations, J. Geodesy, 83, 1031-1040; doi: 10.1007/s00190-009-0321-6.

Abbondanza, C. and P. Sarti (2010). Effects of illumination functions on the computation of gravity-dependent signal path variation models in Cassegrainian VLBI telescopes, J. Geodesy, 84, 515-525; doi: 10.1007/s00190-010-0389-z.

Altamimi, Z., X. Collilieux, J. Legrand, B. Garayt and C. Boucher (2007). ITRF2005: A new release of the International Terrestrial Reference Frame based on time series of station positions and Earth Orientation Parameters, J. Geophys. Res., 112, B09401; doi: 10.1029/2007JB004949.

Altamimi Z. (2010). Quality assessment of the local ties used in the ITRF2008, Geophysical Research Abstracts, 12, EGU2010-4840, EGU General Assembly 2010, 2-7 May 2010, Vienna, Austria.

Angermann, D., M. Seitz and H. Drewes (2010). Analysis of local ties from ITRF2008 computations, Geophysical Research Abstracts, 12, EGU2010-11978, EGU General Assembly 2010, 2-7 May 2010, Vienna, Austria.

Bitelli, G., J. Campbell, M. Negusini, P. Sarti and L. Vittuari (2005). Determination of vertical motion from levelling data in the wider area of Medicina and the Apennine foot-hills, Proc. 17th working meeting on European VLBI for Geodesy and Astrometry, April 22-23, 2005, 
Noto (Italy), 56-63.

Böckmann, S., T. Artz and A. Nothnagel (2010a). VLBI terrestrial reference frame contributions to ITRF2008, J. Geodesy, 84, 201-219; doi: 10.1007/s00190-009-0357-7.

Böckmann, S., T. Artz, A. Nothnagel and V. Tesmer (2010b). International VLBI service for geodesy and astrometry: EOP combination methodology and quality of the combined products, J. Geophys. Res., 115, B04404; doi: 10.1029/2009JB006465.

Campbell, J. and A. Nothnagel (2000). European VLBI for crustal dynamics, J. Geodyn., 30, 321-326.

Carter, E., A.E.E. Rogers, C.C. Counselman and I.I. Shapiro (1980). Comparison of geodetic and radio interferometric measurements of the Haystack-Westford base line vector, J. Geophys. Res., 85, 2685-2687.

Clark, T.A. and P. Thomsen (1988). Deformations in VLBI antennas, Tech report 100696, NASA, Greenbelt, MD, http: / / ntrs.nasa.gov/archive/nasa/ casi.ntrs.nasa.gov/ 19 880009586_1988009586.pdf. Last accessed: 24 May 2010.

Coulot, D., P. Berio, P. Bonnefond, P. Exertier, D. Féraudy, O. Laurain and F. Deleflie (2008). Satellite Laser Ranging Biases and Terrestrial Reference Frame Scale Factor, In: Observing our changing Earth, edited by M.G. Sideris, I.A.G. Symp., 133, 39-46.

Dilssner, F., G. Seeber, G. Wübenna and M. Schmitz (2008). Impact of near-field effects on the GNSS position solution, Proc. 21st International Technical Meeting of the Satellite Division of The Institute of Navigation (ION GNSS 2008), September 16-19, 2008, Savannah, GA, USA, 612-624

Dow, J.M., R.E. Neilan and C. Rizos (2009). The International GNSS Service in a changing landscape of Global Navigation Satellite Systems, J. Geodesy, 83, 191-198; doi: 10.1007/ s00190-008-0300-3.

Fey, A., D. Gordon and C.S. Jacobs (2009). The Second Realization of the International Celestial Reference Frame by Very Long Baseline Interferometry, Presented on behalf of the IERS / IVS Working group, Technical Note 35, Frankfurt am Main: Verlag des Bundesamts für Kartographie und Geodäsie; http://www.iers.org/nn_11216/IERS/EN/Publications/TechnicalNotes/tn35.html. Last accessed: 24 May 2010.

Gendt, G., A. Nothnagel, E. Pavlis, F. Lemoine, T. van Dam and G. Appleby (2007). Technique-specific biases and effects at co-location sites/satellites, In: Unified Analysis Workshop 2007, http://www.iers.org/nn_10902/SharedDocs/Publikationen/EN/IERS/Workshops/UAW PosPap__Session__2__Dec02, templateId=raw, property=publicationFile.pdf/UAW_PosPap_Session_2_De c02.pdf. Last accessed: 24 May 2010.

Kierulf, H.P., B.R. Pettersen, D.S. MacMillan and P. Willis (2009). The kinematics of Ny-Ålesund from space geodetic data, J. Geodyn., 48, 37-46; doi: 10.1016/j.jog.2009.05.002. King, A.M., Z. Altamimi, J. Boehm, M. Bos, R. Dach, P.
Elosegui, F. Fund, M. Hernandez-Pajares, D. Lavallee, P. J. Mendes Cerveira, N. Penna, R.E.M. Riva, P. Steigenberger, T. van Dam, L. Vittuari, S.D.P. Williams and P. Willis (2010). Improved Constraints on Models of Glacial Isostatic Adjustment: A Review of the Contribution of Ground-Based Geodetic Observations, Surv. Geophys.; doi: 10.1007 / s10712-010-9100-4.

Lidberg, M., J.M. Johansson, H.-G. Scherneck and G.A. Milne (2010). Recent results based on continuous GPS observations of the GIA process in Fennoscandia from BIFROST, J. Geodyn., 50, 8-18; doi: 10.1016/j.jog.2009.11.010.

Nothnagel, A. (2008). Conventions on thermal expansion modelling of radio telescopes for geodetic and astrometric VLBI, J. Geodesy, 83, 787-792; doi 10.1007/s00190-0080284-Z.

Petrov, L. (2008). Introduction to VLBI Time Delay (VTD) package; http: / / astrogeo.org/vtd/vtd_01.html. Last accessed: 24 May 2010.

Petrov, L., D. Gordon, J. Gipson, D. MacMillan, C. Ma, E. Fomalont, R.C. Walker and C. Carabajal (2009). Precise geodesy with the very long baseline array, J. Geodesy, 83, 859-876; doi: 10.1007/s00190-009-0304-7.

Robertson, D.S. (1991). Geophysical applications of very-longbaseline interferometry, Rev. Mod. Phys., 63, 899-918.

Ryan, J.W., C. Ma and D.S. Caprette (1993). NASA Space Geodesy Program: GSFC data analysis, 1992. Crustal Dynamics Project VLBI geodetic results, 1979 - 1991, Final Report NASA. Goddard Space Flight Center, Greenbelt, MD; http: / / ntrs.nasa.gov/ archive/ nasa/ casi.ntrs.nasa.gov/ 199 20014993_1992014993.pdf. Last accessed: 24 May 2010.

Sarti, P., P. Sillard and L. Vittuari (2004). Surveying co-located space geodetic instruments for ITRF computation, J. Geodesy, 78, 210-222; doi: 10.1007/ s00190-004-0387-0.

Sarti, P., C. Abbondanza and L. Vittuari (2009a). Gravitydependent signal-path variation in a large VLBI telescope modelled with a combination of surveying methods, J. Geodesy, 83, 1115-1126; doi: 10.1007/s00190-009-0331-4.

Sarti, P., L. Vittuari and C. Abbondanza (2009b). Laser scanner and terrestrial surveying applied to gravitational deformation monitoring of large VLBI telescopes' primary reflector, J. Surv. Eng., 135, 136-148; doi: 10.1061/(ASCE)SU.1943 -5428.0000008 .

Sarti, P., C. Abbondanza, L. Petrov and M. Negusini (2010). Height bias and scale effect induced by antenna gravity deformations in geodetic VLBI data analysis, J. Geodesy; doi: 10.1007 / s00190-010-0410-6.

Schlüter, W. and D. Behrend (2007). The International VLBI Service for Geodesy and Astrometry (IVS): current capabilities and future prospects, J. Geodesy, 81, 379-387; doi: 10.1007/ s00190-006-0131-z.

Schmid R., X. Collilieux, P. Steigenberger and U. Hugentobler (2010). Estimation of GPS satellite antenna z-offsets from reprocessed SINEX files, Geophysical Research Abstracts, 
12, EGU2010-9557, EGU General Assembly 2010, 2-7 May 2010, Vienna, Austria.

Setti, G. (2006). Synthetic history of the SRT project, In: Science with the Sardinia Radio Telescope (Bologna, May 1011, 2005), edited by J. Brand, K.H. Mack and I. Prandoni, Mem. S.A.It., Suppl., 10, 15-18.

Tomasi, P., F. Mantovani, R. Ambrosini, A. Bombonati, G. Grueff, A. Nothnagel and H. Schuh (1988). The first geodetic VLBI experiment with the Bologna radio telescope, Nuovo Cimento C, 11, 205-208.

Tomasi, P. (1993). Noto station status report, In: Proc. 9th Working Meeting on European VLBI for Geodesy and Astrometry, 81, Mitteilungen aus den Geodätischen Instituten der Rheinischen Friedrich-Wilhelms-Universität Bonn, edited by J. Campbell and A. Nothnagel, 11-12.

Tomasi, P., M.J. Rioja and P. Sarti (1999). The European VLBI network activity in geodesy: crustal deformation in Europe, New Astron. Rev., 43, 603-607; doi: 10.1016/S13876473(99)00062-7.

Wessel, P. and W.H.F. Smith (1998). New, improved version of generic mapping tools released, Eos Trans. AGU, 79 (47), 579.

Willis, P., B.J. Haines, and D. Kuang (2007). DORIS satellite phase center determination and consequences on the derived scale of the Terrestrial Reference Frame, Adv. Space Res., 10, 1589-1596.

Zerbini, S., F. Raicich, B. Richter, V. Gorini and M. Errico (2010). Hydrological signals in height and gravity in northeastern Italy inferred from principal components analysis, J. Geodyn., 49, 190-204; doi: 10.1016/j.jog.2009.11.001.

${ }^{\star}$ Corresponding author: Pierguido Sarti,

Istituto di Radioastronomia (IRA) - Istituto Nazionale di Astrofisica (INAF), Bologna, Italy; e-mail: p.sarti@ira.inaf.it.

(C) 2010 by the Istituto Nazionale di Geofisica e Vulcanologia. All rights reserved. 